\title{
Insulation Failure Prediction Model of Power Cable in Fire
}

\author{
Jin-mei $\mathrm{Li}^{1,2}$, Jia-qing Zhang ${ }^{3}$, Qiang $\mathrm{Li}^{1}$ and Shi-jing $\mathrm{Ren}^{1}$ \\ ${ }^{1}$ Chinese People's Armed Police Academy, Department of Fire Protection Engineering, 065000 Langfang, China \\ ${ }^{2}$ University of Science and Technology Beijing, School of Civil and Resource Engineering, 100083 Beijing, China \\ ${ }^{3}$ State Grid Anhui Electric Power Research Institute, 230022 Hefei, China
}

\begin{abstract}
With input parameters being determined, simplified physical model and heat conduction equation were adopted to establish a power cable insulation failure prediction model in fire, and the validation test was carried out. The internal structure of the cable was simplified to a one-dimensional physical model with three layers, to derive the calculation method of internal structure parameters of cable physical model. Differential equation of heat conduction, which could reflect the internal temperature of cable, was constructed based on basic assumptions, to establish a cable insulation cable failure prediction model with mixed layer heat diffusion coefficient, environment temperature change and insulation cable failure temperature as input parameters. The method to determine model input parameters was also proposed. ZR-YJV and SDR-1 cable thermal radiation furnace were selected in the experiments to obtain insulation failure temperature and time, as well as environmental temperature change of the cable. The experimental results showed that the constructed model had relative small error in predicting cable insulation failure time.
\end{abstract}

\section{Introduction}

Cable is the artery and nerve for the equipment to operate, which functions a lot in electric power transfer and information transmission. The function failure of cable is the main reason for disaster expansion. Therefore, for certain places where existing fire risk of cable, it is necessary to conduct research on the failure and risk in fire. And reasonably, the study on the predicting insulating failure model of the cable under the circumstance of fire is the foundation of fire probability safety assessment [1].

The research shows that the insulating failure of cable in fire is relevant to the surrounding radiation heat flux and the temperature, while irrelevant to the number of the cores of cable [2,3]. Multiple-core cable insulating failure usually occurs in the internal side of the shielding layer [4], as the failure point. When the insulating cable fails, the temperature of the failure point is relevant to the thermal properties of the cable material. The failure point temperature of certain material remains in specific temperature range $[5,6]$. Therefore, cable heat transmission model, which predicts the insulating failure time of cable in fire, is usually employed in the predicting insulating failure model of the cable. Based on the chemical properties of cable in fire and the research on the thermal decomposition process of the material inside the cable, Valbuena [7] discusses the ultimate temperature of polymer insulating cable under the condition of function of power supply and establishes the failure probability model of cable. However, the thermal decomposition process of the material inside the cable is extremely complex, which is subjective to multiple elements such as cable form, structure, the thermal properties of the material of insulating shield and surrounding temperature. The Valbuena model is greatly limited in practice. McGrattan [4] puts forward a more applicable predicting model - the THIEF Model, which neglects the complex structure inside the cable and simplify it as a long homogeneous cylinder which possesses certain thermal properties. By establishing unidimensional heat transmission equation of simplified cable, we can obtain the temperature-time changing law of any point within the cable. By setting the failure point and the temperature of it, we can calculate the time for the cable to reach the specific insulating failure temperature in fire. And based on this, Buckno [8] puts forward the cylinder model of cable bunch and rectangle model of cable, which is closely arranged in the form of cable bridge and covers the insulating panel, and improves the THIEF model based on different heat transmission forms and properties under different alignment. Qiang [9] extends the model into the failure prediction of the ignited cable and establishes unidimensional Five-layer heat transmission model. The THIEF Model neglects the structure form inside the cable, which although reduces the difficulty of cable application in practice greatly, but the assumption of setting the surrounding convective heat transfer coefficient as constant is quite different from the actual ambient condition. Meanwhile, although the insulating failure temperature of heat-setting cable and thermoplastic cable is given, it does not involve the YJV 
which is most commonly used in engineering, with its XLPE insulating layer and PVC protective layer.

This article is based on the simplified unidimensional three-layer cable physical model, establishing the differential equation of heat conduction which indicates the temperature distribution inside the cable, proposing the predicting insulating failure model of the cable of which the mixed-layer heat-transmission index, the temperature change of the fire condition and cable insulating failure point serve as the inputting index. This model can predict the insulating failure time of the cable in alterable surrounding temperature, and the cable which is both heat-setting and thermoplastic.

\section{Simplification of physical cable model}

The Fig. 1 is the diagram of representative internal structure of cable. Among the diagram, the insulating layer isolates the core and the ground, as well as the cores themselves electrically, which enables the electric power to transmit reliably; the protecting layer defends the cable against external impurity and hydrosphere, also prevents the outside force from devastate the cable; the filling material decreases the usage of protective material in the squeezing process of the protecting-layer, simultaneously ensures the integrity of the cable.

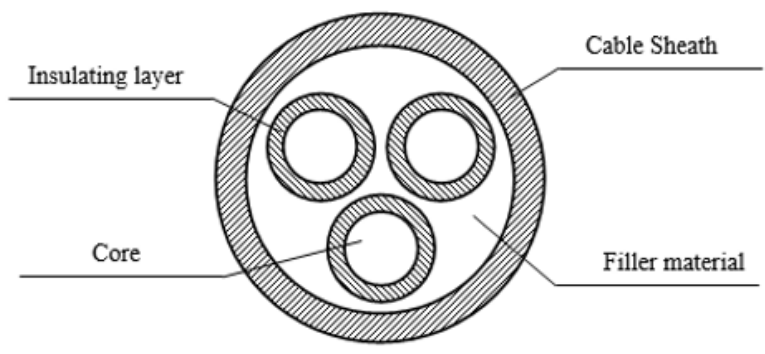

Fig. 1. Diagram of typical cable internal structure.

The heat-transmitting properties of the insulating layer, the protecting layer and the protecting layer mutually determine the internal heat transmission and temperature distribution of the cable under the effect of external heat. To establish the cable heat-transmission equation, we can simplify its internal structure into three concentric circles, as is showed in Fig. 2. The simplified cable physical model is core, insulating layer and mixed layer from inside to outside successively, among which the mixed layer is the simplified substitution for filing material and protective layer, packed outside the insulating layer.

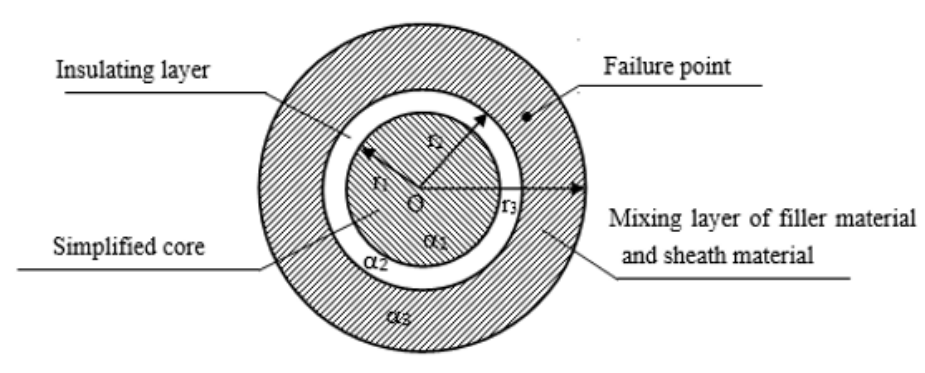

Fig. 2. Simplified cable internal structure.
The internal structure of the simplified cable physical model has following properties:

(1) The external diameter remains the same, that is:

$$
r_{3}=D / 2
$$

Where $r_{3}$ is simplified cable semidiameter, $D$ is primitive cable diameter.

(2) The cable core section proportion equals the total sum of primitive cable core section proportion, that is:

$$
r_{1}=\frac{\sqrt{n}}{2} \cdot d
$$

Where $r_{1}$ is simplified cable core semidiameter, $d$ is primitive cable core diameter, $n$ is the number of primitive cable core.

(3) The thickness of the insulating layer remains the same, also:

$$
r_{2}=r_{1}+l_{\mathrm{i}}
$$

Where $r_{2}$ is the semidiameter of simplified insulating-layer external boundary, $l_{i}$ is primitive thickness of the cable insulating-layer.

(4) Failure point distance, that is:

$$
\lambda=r_{3}-l_{s}
$$

Where $\lambda$ is the distance between simplified insulating failure point and the original point, $l_{\mathrm{s}}$ is the thickness of primitive insulating-layer of the cable.

\section{Predicting model of power cable insulating failure}

\subsection{Fundamental assumption}

Assumption of simplified cable heat-transmission physical model as follow:

(1) Before the cable insulating failure occurs, its physicochemical properties of the internal material remain the same, density of the material $\left(\rho, \mathrm{kg} \cdot \mathrm{m}^{-3}\right)$, specific heat $\left(c, \mathrm{~J} \cdot \mathrm{kg}^{-1} \cdot \mathrm{K}^{-1}\right)$ and thermal conductivity $(k$, $\left.\mathrm{W} \cdot \mathrm{m}^{-1} \cdot \mathrm{K}^{-1}\right)$ are all constant;

(2) The effect of the core heat on the cable heattransmission is neglected;

(3) The external heat transmits along the direction of cable cross section semidiameter;

(4) The burning of the cable occurs after the insulating failure occurs;

(5) If the temperature of the cable insulating failure point reaches the insulating failure temperature, the insulating failure of the cable occurs.

\subsection{Differential equation of heat conduction}

According to the Law of Conservation of Energy and the Law of Fourier, the differential equation of heat conduction, which indicates the temperature distribution inside the cable, is established. As is showed in Fig. 2, 
the cylindrical coordinate system $(r, \phi, z)$ can be represented by:

$$
\rho c \frac{\partial T}{\partial t}=\frac{1}{r} \frac{\partial}{\partial r}\left(k r \frac{\partial T}{\partial r}\right)+\frac{1}{r^{2}} \frac{\partial}{\partial \phi}\left(k \frac{\partial T}{\partial \phi}\right)+\frac{\partial}{\partial z}\left(k \frac{\partial T}{\partial z}\right)+q_{v}
$$

Where $r$ is the distance from along the cable cross section to the circle center, $\mathrm{mm}$. $T$ is the temperature at the distance of $r$ from the circle centre along the cable cross section, ${ }^{\circ} \mathrm{C}$. $t$ is time, s. $\rho$ is the density of the material, $\mathrm{kg} \cdot \mathrm{m}^{-3}$. $c$ is the specific heat of the material, $\mathrm{J} \cdot \mathrm{kg}^{-1} \cdot \mathrm{K}^{-1} \cdot k$ is the heat-transmission index of the material, $\mathrm{W} \cdot \mathrm{m}^{-1} \cdot \mathrm{K}^{-1}$.

According to Assumption (1) and Assumption (2), we simplify the heat-transmission process from the external heat along the circle cross section into unidimensional unsteady heat-transmission process without participation of internal heat source, and obtain the differential equation of heat conduction of the cable heat-transmission physical model according to Eq. 5:

$$
\rho c \frac{\partial T}{\partial t}=\frac{1}{r} \frac{\partial}{\partial r}\left(k r \frac{\partial T}{\partial r}\right)
$$

No fire, the initial condition of the differential equation of heat conduction is:

$$
\left\{\begin{array}{l}
t=0 \\
T=T_{0} \\
0 \leq r \leq R
\end{array}\right.
$$

Where $T_{0}$ is initial surrounding temperature, ${ }^{\circ} \mathrm{C} . R$ is cable semidiameter, $\mathrm{mm}$.

When the cable is exposed to fire on condition of turbulent and burning smoke, the right-boundary condition of the differential equation of heat conduction is:

$$
\left\{\begin{array}{l}
r=R \\
T=T_{s}
\end{array}\right.
$$

Where $T_{\mathrm{s}}$ is the surrounding burning smoke temperature, ${ }^{\circ} \mathrm{C}$.

Considering that the core of the cable possesses great heat-transmission property, the required time for the burning-smoke surrounding to the internal cable is much longer than that along the cable core. Therefore, it is possible to assume that there is no temperature gradient from the cable core along the circle cross section, that is to say, the left-boundary condition of the differential equation of heat conduction is:

$$
\left.\frac{\partial T}{\partial r}\right|_{0 \leq r \leq r_{1}}=0
$$

\subsection{Numerical solution and input parameters}

Partial differential equation is usually difficult to analyses numerically. Therefore, the finite difference method is employed to obtain the numerical solution of differential equation of heat conduction of the cable heat-transmission physical model.
As is showed in Eq. 6, the differential equation of heat conduction of the cable heat-transmission physical model is unidimensional linear equation, which complies with parabolic partial differential equation. Therefore, the Crank-Nicolson solution is employed to conduct finite difference calculation to the heat-transmission differential equation, and reasonably the calculation formula of the predicting cable insulating failure model is showed as follows:

$$
\begin{aligned}
\rho c \frac{T_{i}^{n+1}-T_{i}^{n}}{\delta t}= & \frac{k}{r_{i+1}+r_{i}}\left[r_{i} \frac{T_{i+1}^{n}-T_{i}^{n}}{(\delta r)^{2}}-r_{i-1} \frac{T_{i}^{n}-T_{i-1}^{n}}{(\delta r)^{2}}\right] \\
& +\frac{k}{r_{i+1}+r_{i}}\left[r_{i} \frac{T_{i+1}^{n+1}-T_{i}^{n+1}}{(\delta r)^{2}}-r_{i-1} \frac{T_{i}^{n+1}-T_{i-1}^{n+1}}{(\delta r)^{2}}\right]
\end{aligned}
$$

Where $\delta_{\mathrm{r}}$ is the dimensional step along the cable semidiameter. When the semidiameter of the cable is equally divided into $N$ sections, $\delta_{\mathrm{r}}=D /(2 N)$ (In the study, it is assumed as $0.1 \mathrm{~mm})$, and $r_{0}=0, r_{\mathrm{N}}=D / 2 . \delta_{\mathrm{t}}$ is time step, and $\delta_{\mathrm{t}}=c \cdot \rho \cdot \delta_{\mathrm{r}}^{2} /(2 \mathrm{k})$; Tin is the temperature of the $n$ th time step and the $i$-th dimensional step, ${ }^{\circ} \mathrm{C}$.

The input parameters of the predicting insulating failure model of the cable includes:

(1) The insulating failure temperature of the cable. It should be obtained by experiment as it differs a lot among different cable forms;

(2) The heat-extending parameter of each layer heatconducting medium of the cable model. Among this, the parameter of the cable core and the insulating layer can be referred to the thermal physical parameter of the material itself while the parameter of the mixed layer should be obtain by experiment;

(3) Cable internal structure parameter can be obtained by measuring cable internal structure parameter, and calculating according to Eq. 1 Eq. 4;

(4) The ambient temperature change process in fire. To simplify the calculation, the ambient temperature- time curve should be linearly fitted according to the certain surrounding, and the fitted curve should be regarded as inputting parameter.

\section{Experimental setup}

\subsection{Cable thermal radiation experimental furnace}

The structure of SDR-1 Type cable thermal radiation experimental furnace is showed in Fig. 3.

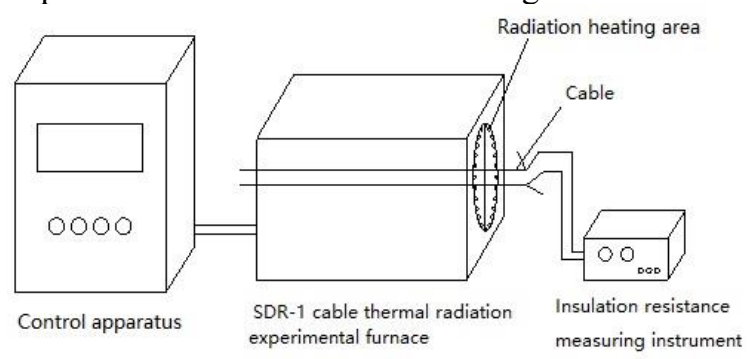

Fig. 3. Framework of SDR-1 cable thermal radiation experimental furnace. 
The external size of the experiment oven is 80 $\mathrm{cm}(\mathrm{L}) \times 75 \mathrm{~cm}(\mathrm{~W}) \times 81 \mathrm{~cm}(\mathrm{H})$. The furnace chamber is cylindrical (section diameter is $33 \mathrm{~cm}$ ), along whose in wall aligned homogeneously 24 infrared radiation heating pipes with rated output power $2.5 \mathrm{~kW}$ each (As is showed in Fig. 4). The output power of the infrared radiation heating pipes can be set by controlling equipment to simulate the heating condition of the cable in fire. The experiment selected $3.0 \mathrm{~kW} / \mathrm{m}^{2}, 3.6 \mathrm{~kW} / \mathrm{m}^{2}$ and $4.2 \mathrm{~kW} / \mathrm{m}^{2}$ as radiation heat flux. The experimental scene is showed in Table 1 .

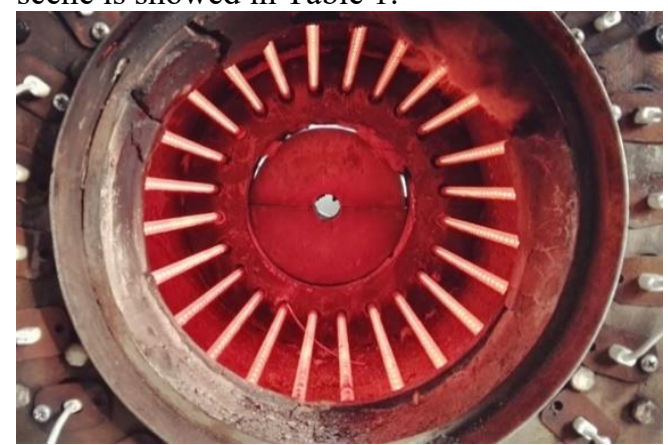

Fig. 4. Chamber of cable thermal radiation experimental furnace.

In this experiment, we chose the TH2683A insulating resistance tester to measure the insulating states between the cables. In the experiment, the output power in the testing side of the tester is $\mathrm{DC} 1000 \mathrm{~V}$. The furnace chamber temperature, cable outside surface temperature and cable internal temperature (failure point temperature) were tested by thermocouple. The chosen thermocouple is K-Type sheathed thermocouple with its $1 \mathrm{~mm}$ diameter and the data collection was conducted by RIGOL-M300 data collector.

The tested cable processing method can be reviewed in Reference [10], the judging standard for cable insulating failure can be reviewed in Reference [11].

\subsection{Cables}

The experiment selected ZR-YJV Cable. In order to expand the practical value of the research conclusion, we took the effect from core quantity and conductor cross section proportion on the result into account when we chose the cable type. The tested cable is showed in Table 1 , which covered two-core, three-core, four-core and five-core, with conductor cross section proportion 2.5 $\mathrm{mm}^{2}, 4 \mathrm{~mm}^{2}, 6 \mathrm{~mm}^{2}$ and $10 \mathrm{~mm}^{2}$ each.

\subsection{Experimental Results}

Under external heat radiation effect, the changing law of the ambient temperature (furnace chamber temperature), failure point temperature (cable internal temperature) and the representative insulating resistance between the cables are showed in Fig. 5.

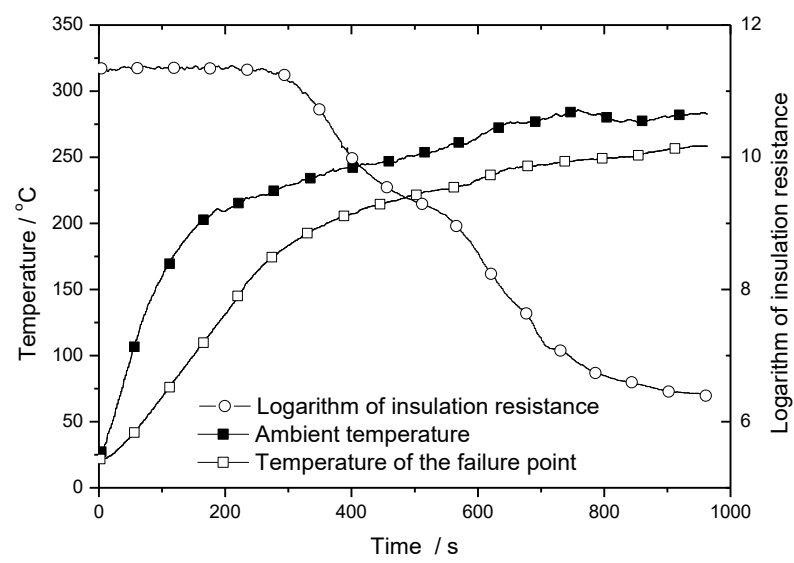

Fig. 5. Experimental result of ZR-YJV-3×4 $\left(q "=3.0 \mathrm{~kW} / \mathrm{m}^{2}\right)$.

By recording the time at which the cable insulating failure occurred, we obtained the insulating failure temperature in all experimental circumstances, as is showed in Table 1.

Table 1. Experimental results and computing results.

\begin{tabular}{|c|c|c|c|c|c|c|c|c|c|c|c|c|c|}
\hline \multirow{4}{*}{ No. } & \multirow{4}{*}{$\begin{array}{c}\text { Cable } \\
\text { type }\end{array}$} & \multicolumn{12}{|c|}{ Radiation heat flux $\left(\mathrm{kW} / \mathrm{m}^{2}\right)$} \\
\hline & & \multicolumn{4}{|c|}{3.0} & \multicolumn{4}{|c|}{3.6} & \multicolumn{4}{|c|}{4.2} \\
\hline & & \multirow{2}{*}{$\begin{array}{l}\text { Insulating } \\
\text { failure } \\
\text { temperature } /{ }^{\circ} \mathrm{C}\end{array}$} & \multicolumn{3}{|c|}{ Insulating Failure Time /s } & \multirow{2}{*}{$\begin{array}{l}\text { Insulating Failure } \\
\text { Temperature } /{ }^{\circ} \mathrm{C}\end{array}$} & \multicolumn{3}{|c|}{ Insulating Failure Time /s } & \multirow{2}{*}{$\begin{array}{c}\text { Insulating } \\
\text { Failure } \\
\text { Temperature } \\
\quad{ }^{\circ} \mathrm{C}\end{array}$} & \multicolumn{3}{|c|}{ Insulating Failure Time $/ \mathrm{s}$} \\
\hline & & & $\mathrm{ER}^{\mathrm{a}}$ & $\mathrm{CR}^{\mathrm{b}}$ & RE $/ \%$ & & $\mathrm{ER}^{\mathrm{a}}$ & $\mathrm{CR}^{\mathrm{b}}$ & $\mathrm{RE} / \%$ & & $\mathrm{ER}^{\mathrm{a}}$ & $\mathrm{CR}^{\mathrm{b}}$ & RE $/ \%$ \\
\hline 1 & $2 \times 6$ & 235.21 & 580 & 612 & 5.52 & 230.62 & 571 & 558 & 2.28 & 255.39 & 483 & 490 & 1.45 \\
\hline 2 & $3 \times 2.5$ & 229.86 & 555 & 546 & 1.62 & 232.09 & 424 & 461 & 8.73 & 242.33 & 464 & 439 & 5.39 \\
\hline 3 & $3 \times 4$ & 236.78 & 617 & 587 & 4.86 & 230.79 & 397 & 498 & 25.44 & 229.61 & 480 & 452 & 5.83 \\
\hline 4 & $3 \times 6$ & 228.63 & 638 & 664 & 4.08 & 226.75 & 553 & 539 & 2.53 & 238.24 & 497 & 491 & 1.21 \\
\hline 5 & $3 \times 10$ & 222.07 & 783 & 769 & 1.82 & 234.12 & 506 & 601 & 18.77 & 231.91 & 634 & 585 & 7.73 \\
\hline 6 & $4 \times 6$ & 231.63 & 643 & 637 & 0.93 & 217.13 & 589 & 585 & 0.68 & 239.66 & 533 & 511 & 4.13 \\
\hline 7 & $5 \times 6$ & 232.14 & 693 & 718 & 3.61 & 229.21 & 649 & 636 & 2.00 & 236.66 & 583 & 557 & 4.46 \\
\hline 1 & $2 \times 6$ & 235.21 & 580 & 612 & 5.52 & 230.62 & 571 & 558 & 2.28 & 255.39 & 483 & 490 & 1.45 \\
\hline
\end{tabular}

${ }^{\mathrm{a}}$ Experimental result

${ }^{\mathrm{b}}$ Computing result 


\section{Verification of the predicting model}

\subsection{Input parameters of predicting model}

\subsubsection{Insulating failure temperature}

Fig. 6 is the cable insulating failure temperature in all experimental conditions. It can be concluded that the ambient radiation heat flux and cable type had little effect on cable insulating failure temperature. The highest insulating failure temperature is $255.39^{\circ} \mathrm{C}$, while the lowest is $217.13^{\circ} \mathrm{C}$.

The research showed that the insulating temperature stays mainly the same for the same kind of insulating cable material. Therefore, when predicting model was calculated, we should set the average insulating failure temperature $\left(233^{\circ} \mathrm{C}\right)$ as the judging standard for cable insulating failure.

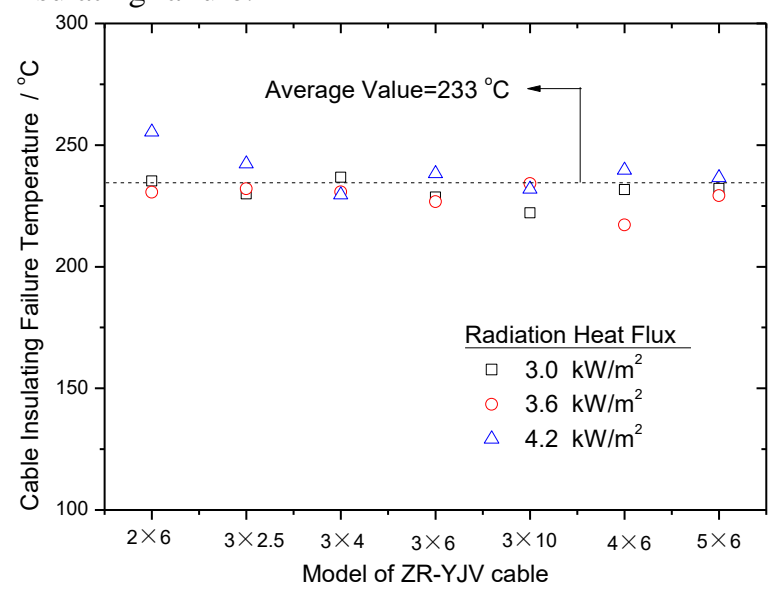

Fig. 6. Insulation failure temperature of ZR-YJV.

\subsubsection{Thermal diffusivity of mixing layer}

To fit the temperature curve of the failure point, we can obtain the thermal diffusivity of the mixed layer in simplified cable model in all experimental conditions, as is showed in Fig. 7.

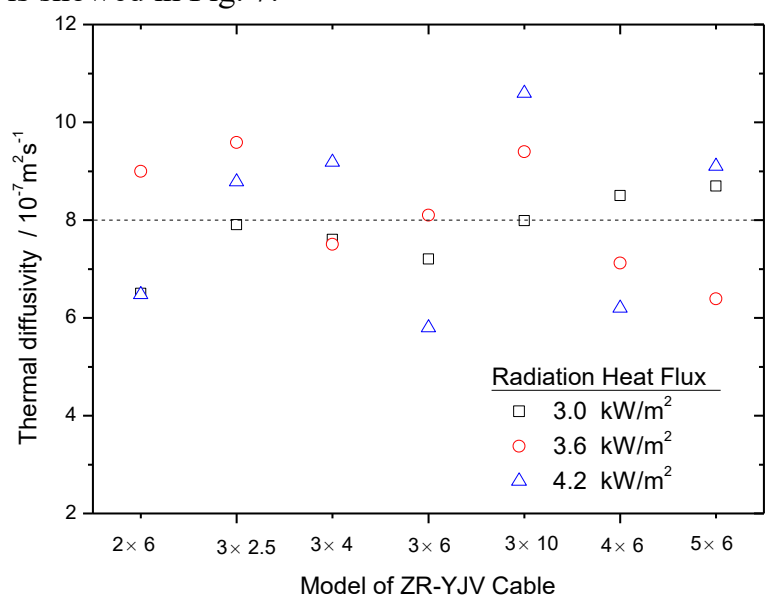

Fig. 7. Thermal diffusivity of mixing layer of simplified cable model.
When conducting the calculation, we set $8.00 \times 10^{-7}$ $\mathrm{m}^{2} / \mathrm{s}$ as the thermal diffusivity of filling material and protecting layer in which the ZR-YJV Cable Model was used. Thermal diffusivity of other material was showed in Table 2.

Table 2. Thermophysical parameters values of ZR-YJV.

\begin{tabular}{|c|c|c|}
\hline $\begin{array}{c}\text { Cable } \\
\text { construction }\end{array}$ & $\begin{array}{c}\text { Heat-conducting } \\
\text { medium of cable }\end{array}$ & $\begin{array}{c}\text { Thermal } \\
\text { diffusivity } / \mathbf{m}^{\mathbf{2}} \mathbf{~ s}^{-\mathbf{1}}\end{array}$ \\
\hline Copper core & Copper & $1.16 \times 10^{-4}$ \\
\hline Insulating layer & $\begin{array}{c}\text { Crosslinked } \\
\text { polyethylene }\end{array}$ & $1.52 \times 10^{-7}$ \\
\hline Mixing layer & $\begin{array}{c}\text { Filler material and } \\
\text { sheath material }\end{array}$ & $8.00 \times 10^{-7}$ \\
\hline
\end{tabular}

\subsubsection{Internal Structure Parameters of Simplified Cable Model}

The internal structure of experimental cable is showed in Table 3. According to Eq. $1 \sim$ Eq. 4, we could calculate the internal structure index of the simplified cable model, as is showed in Table 4.

Table 3. Internal structure parameters of ZR-YJV cables.

\begin{tabular}{|c|c|c|c|c|c|}
\hline \multirow{2}{*}{ No. } & \multirow{2}{*}{$\begin{array}{c}\text { Cable } \\
\text { type }\end{array}$} & \multicolumn{2}{|c|}{$\begin{array}{c}\text { Diameter } \\
\text { /mm }\end{array}$} & \multicolumn{2}{c|}{$\begin{array}{c}\text { Thickness } \\
\text { /mm }\end{array}$} \\
\cline { 3 - 6 } & & Cable & $\begin{array}{c}\text { Core of } \\
\text { cable }\end{array}$ & $\begin{array}{c}\text { Insulation } \\
\text { layer }\end{array}$ & $\begin{array}{c}\text { Sheath } \\
\text { layer }\end{array}$ \\
\hline 1 & $2 \times 6$ & 14.26 & 2.76 & 1.30 & 1.58 \\
\hline 2 & $3 \times 2.5$ & 10.34 & 2.00 & 0.66 & 1.28 \\
\hline 3 & $3 \times 4$ & 10.38 & 2.02 & 0.86 & 1.30 \\
\hline 4 & $3 \times 6$ & 14.22 & 2.76 & 0.98 & 1.30 \\
\hline 5 & $3 \times 10$ & 16.68 & 3.70 & 1.14 & 1.90 \\
\hline 6 & $4 \times 6$ & 15.58 & 2.66 & 0.90 & 1.60 \\
\hline 7 & $5 \times 6$ & 16.04 & 2.56 & 0.90 & 1.64 \\
\hline
\end{tabular}

Table 4. Internal structure parameters of simplified cable models.

\begin{tabular}{|c|c|c|c|c|c|}
\hline \multirow{2}{*}{ No. } & \multirow{2}{*}{ Cable type } & \multicolumn{4}{|c|}{ Dimension $/ \mathbf{m m}$} \\
\cline { 3 - 6 } & & $\boldsymbol{r}_{\mathbf{1}}$ & $\boldsymbol{r}_{\mathbf{2}}$ & $\boldsymbol{r}_{\mathbf{3}}$ & $\boldsymbol{r}_{\mathbf{4}}$ \\
\hline 1 & $2 \times 6$ & 1.952 & 3.252 & 7.130 & 5.55 \\
\hline 2 & $3 \times 2.5$ & 1.732 & 2.392 & 5.170 & 3.89 \\
\hline 3 & $3 \times 4$ & 1.749 & 2.609 & 5.190 & 3.89 \\
\hline 4 & $3 \times 6$ & 2.390 & 3.370 & 7.110 & 5.81 \\
\hline 5 & $3 \times 10$ & 3.204 & 4.344 & 8.340 & 6.44 \\
\hline 6 & $4 \times 6$ & 2.660 & 3.560 & 7.790 & 6.19 \\
\hline 7 & $5 \times 6$ & 2.862 & 3.762 & 8.020 & 6.38 \\
\hline
\end{tabular}

\subsubsection{Temperature-time curve in simulating fire}

We linearly fitted the ambient temperature-time curve in different radiation heat flux. The functional formula for fitted curve is showed in Table 5. 
Table 5. Fitting function expressions of temperature - time curve under different experimental situations.

\begin{tabular}{|c|c|c|}
\hline $\begin{array}{c}\text { Radiation heat } \\
\text { flux } / \mathbf{k W} \cdot \mathbf{m}^{-2}\end{array}$ & $\begin{array}{c}\text { Fitting function } \\
\text { expressions }\end{array}$ & $\begin{array}{c}\text { Correlation } \\
\text { coefficient, } \boldsymbol{R}^{\mathbf{2}}\end{array}$ \\
\hline 3.0 & $T=65 \ln (t+6)-140$ & 1.952 \\
\hline 3.6 & $T=72 \ln (t+7)-157$ & 1.732 \\
\hline 4.2 & $T=79 \ln (t+6)-173$ & 1.749 \\
\hline
\end{tabular}

\subsection{Calculation results of predicting model}

Take ZR-YJV-5 $\times 6$ Type Cable, with the ambient radiation heat flux was $4.2 \mathrm{~kW} / \mathrm{m} 2$ as an example, the predicting model calculation result is showed in Fig. 8.

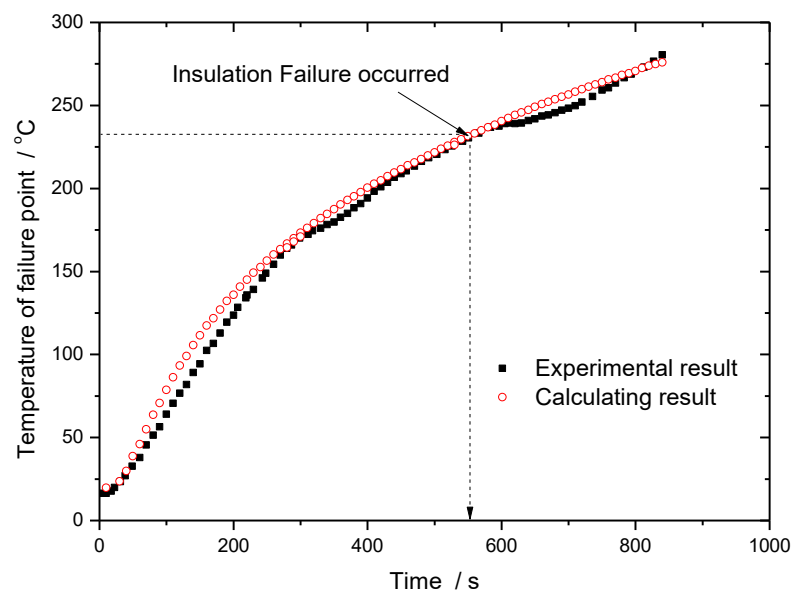

Fig. 8. Comparison between model calculation and experimental result (at insulation failure point).

Setting the judging standard for cable insulating failure as $233^{\circ} \mathrm{C}$, the cable insulating failure time calculated by models in all experiment surroundings is showed in Table 1. The proportional error of insulating time calculated by model is relatively small, which was actually smaller than $10 \%$. By comparing and analyzing the data in which the proportional error was relatively large in Table 1, some large calculated proportional error had bearing on the randomness of the experiment measured result.

\section{Conclusion}

This article puts forward the predicting method for cable insulating failure in fire, based on the engineering requirement of precise prediction of cable insulating failure time in which cable is employed under the condition of fire. It also sets the unidimensional threelayer simplified cable physical model as the foundation, proposes the predicting model for cable insulating failure by establishing the heat-transmission differential equation which indicates the temperature distribution within the cable. Then, it comes up with the judging standard of inputting index for cable insulating failure temperature, the thermal diffusivity of cable heat-conducting medium, the structure index in the simplified cable model and the ambient temperature change process in fire. Finally, by testing the ZR-YJV type cable in SDR-1 Cable Thermal Radiation Experimental Furnace, it obtains the cable insulating failure temperature, insulating failure time and ambient temperature change process. The experimental result shows that the proportional error of insulating failure time calculated by predicting model is relatively small (smaller than 10\%), which basically meets the demand of the safety and risk analysis for fire probability where the cable is employed.

\section{References}

1. Kassawara R P., Hyslop J.S., NUREG/CR-6850 (2005)

2. Murphy J.E., Determination of failure criteria for electric cables exposed to fire for use in a nuclear power plant risk analysis, Worcester Polytechnic Institute, U.S. (2004)

3. Iqbal N., Salley M.H., NUREG-1805 (2004)

4. Mcgrattan K., NUREG/CR-6931, 3 (2007)

5. Nowlen S.P., Wyant F.J., NUREG/CR-6931 1 (2008)

6. Nowlen S.P., Wyant F.J., NUREG/CR-6931 2 (2008)

7. Valbuena G.R., Probabilistic models to estimate fireinduced cable damage at nuclear power plants, University of Maryland, U.S. (2007)

8. Bucknor M.D., Modeling of electrical cable failure in a dynamic assessment of fire risk, The Ohio State University, U.S., (2013)

9. Fu Q., Study on fire performance of typical cables," University of Science and Technology of China, Hefei (2012)

10. Wang G., Experimental study on the effect of fire retardant coating for insulation failure of cable, The Chinese People's Armed Police Academy, Langfang (2011)

11. Yang Y.C., Li Q., Zhang J.Q., Fan M.H., Procedia Engineering, 135 (2016) 